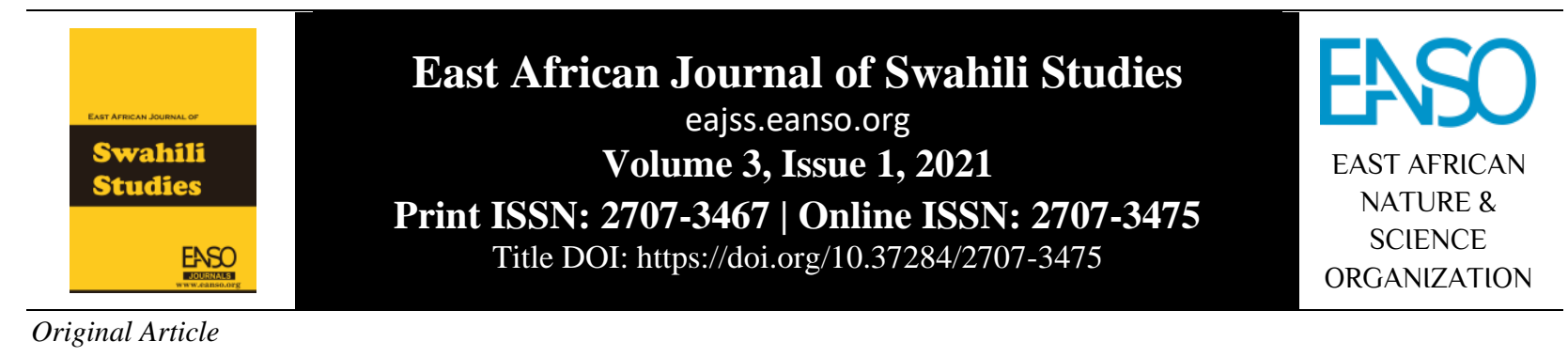

\title{
Urudiaji wa Kisarufi Katika Utenzi wa Seyidina Ali na Mudhari Bin Darimi.
}

\author{
Aloo Ronald Odhiambo ${ }^{1}{ }^{*}$ Prof. Kineene wa Mutiso, PhD $^{1}$ \\ ${ }^{1}$ Chuo Kikuu cha Nairobi. S.L.P 856-01000, Thika, Kenya. \\ *Barua pepe ya mawasiliano: alooronald@gmail.com.
}

DOI ya Nakala: https://doi.org/10.37284/eajss.3.1.404

\section{Tarehe ya Uchapishaji: IKISIRI}

08 Septemba 2021 Katika makala haya, tumechunguza aina na mchango wa urudiaji wa kisarufi katika Utenzi wa Seyidina Ali na Mudhari bin Darimi. Ili kufanikisha hili, Maneno Muhimu: tumeongozwa na nadharia ya Umtindo ambayo inachunguza na kutoa fasiri kwa kazi za isimu na fasihi. Hii ni kwa sababu uchunguzi wa vipengele vya

Urudiaji, kifasihi hauwezi kujisimamia. Nadharia ya Umtindo inahusishwa na Geoffrey Usambamba, Leech katika mwaka wa 1969 kutokana na kazi yake ya kwanza ya ushairi Antistrofia, alioandika huku akizingatia mawazo ya nadharia hii. Lengo la uhakiki wetu ni Epimoni, kuweka wazi aina mbalimbali za urudiaji wa kisarufi zilizotumiwa katika Haipozesia. utungo huu. Baada ya uhakiki wetu, tumegundua kuwa urudiaji wa kisarufi unachangia maudhui, wahusika, vipengele vya kimtindo na vipengele vingine vya fani kwa ujumla katika utungo huu. Isitoshe, urudiaji huu wa kisarufi umekuwa na umuhimu katika kuleta mwangwi na usisitizaji. Tumetumia maelezo na ufafanuzi kutoka katika Utenzi wa Seyidina Ali na Mudhari bin Darimi kama njia ya kubainisha haya.

\section{APA CITATION}

Odhiambo, A. R., \& wa Mutiso, K. (2021). Urudiaji wa Kisarufi Katika Utenzi wa Seyidina Ali na Mudhari Bin Darimi. East African Journal of Swahili Studies, 3(1), 100-107. https://doi.org/10.37284/eajss.3.1.404

\section{CHICAGO CITATION}

Odhiambo, Aloo Ronald, and Kineene wa Mutiso. 2021. "Urudiaji wa Kisarufi Katika Utenzi wa Seyidina Ali na Mudhari Bin Darimi”. East African Journal of Swahili Studies 3(1), 100-107. https://doi.org/10.37284/eajss.3.1.404.

\section{HARVARD CITATION}

Odhiambo, A. R., \& wa Mutiso, K. (2021) "Urudiaji wa Kisarufi Katika Utenzi wa Seyidina Ali na Mudhari Bin Darimi”, East African Journal of Swahili Studies, 3(1), pp. 100-107. doi: 10.37284/eajss.3.1.404.

\section{IEEE CITATION}

$100 \mid$ This work is licensed under a Creative Commons Attribution 4.0 International License. 
A. R. Odhiambo, \& K. wa Mutiso. "Urudiaji wa Kisarufi Katika Utenzi wa Seyidina Ali na Mudhari Bin Darimi”, EAJSS, vol.

3, no. 1, pp. 100-107, Sep. 2021

\section{MLA CITATION}

Odhiambo, Aloo Ronald, \& Kineene wa Mutiso. "Urudiaji wa Kisarufi Katika Utenzi wa Seyidina Ali na Mudhari Bin Darimi”. East African Journal of Swahili Studies, Vol. 3, no. 1, Sep 2021, pp. 100-107, doi:10.37284/eajss.3.1.404.

\section{UTANGULIZI}

Urudiaji ni mbinu ya kifasihi ambayo inahusu kurudiwa kwa maneno yale yale au vifungu vya maneno mara chache ili wazo liweze kuwa wazi na tena likumbukwe zaidi. Mtunzi anapotumia urudiaji katika tenzi za Kiswahili, basi anafanikisha fani na maudhui katika utenzi huo. Hii ni kwa sababu mbinu ya urudiaji ni mojawapo ya mbinu ambazo zinatumiwa na mtunzi kuleta mshikamano katika utungo. Urudiaji wa neno au kifungu cha maneno unaotumiwa na mtunzi wakati mwingine kuongeza msisitizo au kuboresha au kuimarisha ushawishi kuhusu jambo katika utenzi huongeza nguvu kwa yale yanayozungumziwa. Zaidi ya hayo, urudiaji unaweza kutumiwa kuafikia upeo wa utenzi. Matumizi ya urudiaji katika kazi unaleta ukweli ambao ndio mahitaji ya kimsingi ya mitindo, Croft na Robert (2000:57).

Urudiaji wa kisarufi ni mbinu ya kimitindo inayotumiwa ili kuongeza msisitizo na vilevile kuonyesha sifa muhimu za utungo kama vile fani na maudhui. Urudiaji wa kisarufi huwa unatokea wakati sifa mbalimbali za kisarufi kama vile aina ya maneno, wakati na hali, nambari, jinsia na sauti zinaporudiwa na mtunzi. Urudiaji wa kisarufi ni wa umuhimu kwa sababu unawasaidia wachambuzi katika kutathmini na kutoa maoni juu ya mtindo wa utenzi.

\section{Maelezo Mafupi Kuhusu Utenzi wa Seyidina Ali na Mudhari bin Darimi}

Utenzi wa Seyidina Ali na Mudhari bin Darimi (Mhariri Knappert) una beti mia saba sabini na sita (776). Katika beti hizi, mtunzi amezungumzia masuala mbalimbali kama vile suala la uumbaji ambapo mtunzi anasema kuwa Mungu aliumba vitu vingi ila vinne tu ndivyo vitakatifu. Vitu hivi ni pamoja na malaika, milima, siku na hata wanawake. Katika utenzi huu, mtunzi vilevile anazungumzia maswali ishirini yanayoulizwa na Mudhari bin Darimi kwa Sheikh Ali. Sheikh Ali kama mtetezi wa dini ya Kiislamu, anapata ufunuo na kuyajibu maswali hayo vilivyo. Maswali haya, ndiyo misingi ya hukumu Siku ya Kiyama. Aidha, Muislamu anashauriwa na mtunzi kuyazingatia mambo haya kwa sababu ndiyo chanzo cha Uislamu. Maswali haya yanaangazia mambo mbalimbali kuhusu dini. Kulingana na malengo yetu ya utafiti, tumejikita katika kuchunguza namna ambavyo mtunzi anavyotumia urudiaji wa kisarufi katika kuwasilisha fani na maudhui mbalimbali kama vile maudhui ya uumbaji, dini, toba na kadhalika.

\section{Misingi ya Nadharia ya Umtindo}

Mtindo unarejelea namna ambavyo mtunzi anavyotumia lugha yake kulingana na muktadha ili kuwasilisha ujumbe. Kwa mujibu wa Abrams (2015:192), nadharia ya Umitindo ni nadharia inayochunguza sifa za kileksika, sifa za kisarufi (kisintaksia na mofolojia), lugha ya kitamathali na zile za kifonolojia katika kazi. Nadharia hii ilitokana na nadharia ya Balagha na kwa sasa inajisimamia na hata kutumiwa katika uhakiki mbalimbali katika ushairi. Geoffrey Leech (1969) ni mtaalamu wa kwanza aliyetumia nadharia hii katika ushairi. Leeche (1969:2) anakariri kuwa nadharia hii inatumiwa kuhakiki na kutoa fasiri kwa kazi za kifasihi na pia zile za isimu kwa sababu vipengele hivi haviwezi kujisimamia. Aidha, ni vigumu sana kufafanua na kuainisha vipengele vya kifasihi kama vile urudiaji na usambamba bila kurejelea vipengele mbalimbali vya kiisimu.

Leech anaendeleza mjadala huu kwa kusisitiza kuwa nadharia ya Umitindo inasaidia katika kubainisha sheria ambazo zinatumiwa kuteua maneno maalum katika lugha ambayo inatumiwa kutekeleza shughuli tofauti tofauti za jamii zinazouhusisha utoaji na upokezi wa maana ya ujumbe na hata uchanganuzi wa diskosi na fasihi.

Hivyo basi, katika utenzi, mtunzi ana idhini ya kutumia anafora (matumizi ya maneno au virai fulani maalum kuanza mishororo au mistari mbalimbali ya utenzi wake), vilevile mtunzi anaweza kutumia epifora (urudiaji wa virai au 
maneno katika sehemu ya mwisho ya mshororo, virai au sentensi) ili kupitisha ujumbe wake kwenye utungo. Isitoshe, mtunzi anaweza kutumia pilosi (urudiaji wa neno maalum ili kutoa maana mpya).

Nadharia ya Umtindo huchunguza kisemwacho na namna ambavyo kinavyosemwa. Wazo hili limetusaidia katika kuchunguza namna ambavyo mtunzi alivyotumia urudiaji wa kisarufi (mofolojia, sintaksia na usambamba) kama njia ya kufanikisha fani na maudhui katika Utenzi wa Seyidina Ali na Mudhari bin Darimi. Wamitila (2003:39) anaendeleza mjadala huu kwa kusema kuwa Umtindo ni nadharia inayotumiwa kuchunguza vile matukio tofauti tofauti yanavyoelezwa katika isimu na fasihi kwa kutumia lugha.

Katika sehemu hii tumechunguza kipengele cha uchimuzi na mtindo. Kipengele hiki kinashughulikia namna ambavyo mtunzi amekiusha lugha yake kwa kutumia urudiaji wa kisarufi ili kuweka wazi ujumbe wake katika Utenzi wa Seyidina Ali na Mudhari bin Darimi kama njia ya kufanikisha fani na maudhui kwenye utungo huu.

Baadhi ya mihimili ya Umtindo itakayotusaidia katika utafiti wetu ni kama vile: Ujumbe katika kazi ya kifasihi hutokea katika mtindo wa urudiaji wa kisarufi unaohusika. Vipengele vya lugha kama vile mbinu ya urudiaji wa kisarufi na ujumbe vina uhusiano mkubwa na haviwezi vikatenganishwa. Mihimili hii miwili imetusaidia katika kutambua namna ambavyo aina mbalimbali za urudiaji wa kisarufi umetumiwa na mtunzi. Vilevile tumeweza kubainisha namna ambavyo urudiaji huu wa kisarufi unavyouhusiana na ujumbe na kujenga fani na maudhui.

\section{Uchanganuzi}

Mtunzi wa Utenzi wa Seyidina Ali na Mudhari bin Darimi ametumia aina mbalimbali za urudiaji wa kisarufi katika kuangazia masuala tofauti tofauti kwenye utungo wake. Tumebainisha jinsi mtunzi huyu alivyotumia urudiaji wa kisarufi wa aina mbalimbali kama mbinu ya kufanikisha fani na maudhui. Isitoshe, mbinu hii ya urudiaji wa kisarufi ndiyo msingi wa kuelewa ujumbe wa utenzi huu kuhusu Siku ya Kiyama na masuala ya uumbaji.

\section{Urudiaji wa Nambari}

Wamutiso (2014:11) anasema kuwa nambaria zinazotumiwa katika jamii zina ishara tofauti tofauti zinazoleta maana yenye athari katika maisha ya binadamu. Aidha, nambari hizi huleta usufi, uajabu na utakatifu wa kile kinachozungumziwa. Katika Utenzi wa Seyidina Ali na Mudhari bin Darimi, mtunzi anazungumzia suala la uumbaji. Katika ubeti wa 33 hadi 63, mtunzi anasimulia kuwa Mungu aliumba vitu vingi ila vinne pekee ndivyo vitakatifu. Vitu hivi vinne vitakatifu ni pamoja na; malaika wanne wenye kheri (Jiburili, Mikaili, Isirafili na Aziraili), mitume wanne (Iburahimu, Musa, Isa na Muhammadi), majabali manne (Kafu, Turu, Huda na Arafiya), miezi minne mitakatifu (Muharamu, Rajabu Muhhashamu, Shaabani na Ramadhani), siku nne takatifu (Juma, Idi mbili na Arafa) na wanawake wanne wateule (Amina, Sara Mariamu na Saidatina). Masimulizi haya yanaleta unajimi na uaguzi katika utenzi huu. Isitoshe, urudiaji wa nambari nne unachangia ukamilifu wa uumbaji katika dini ya Kiislamu.

\section{Urudiaji wa Mzizi}

Urudiaji huu hutokea wakati mtunzi anaporudia mofimu. Sehemu ya neno linalorudiwa mara nyingi hukaa peke yake kama neno kamili. Katika Utenzi wa Seyidina Ali na Mudhari bin Darimi, urudiaji wa mzizi umetokea kwa njia mbalimbali. Mifano ni kama ifuatayo; mzizi -tu- umerudiwa kwa wingi. Mzizi huu unawakilisha maneno kama vile; watu, mtu na mtuye. Maneno haya yametumiwa na mtunzi ili kuwarejelea waja na hatima yao Siku ya Kiyama. Vilevile kupitia kwa mzizi huu mtunzi analeta msisitizo kwa mambo anayoyasema katika utungo.

\section{Urudiaji wa Umbo la Kitenzi}

Katika utungo huu umbo la kitenzi sikia limerudiwa sana na mtunzi. Umbo hili limerudiwa ili kusisitiza ujumbe wa utenzi kwa Waislamu. Mtunzi anawasisitizia Waislamu kuwa ni vyema wayasikilize yale wanayofundishwa kama njia ya pekee ya kujiepusha na maangamio ya Jehanamu. Kitenzi hiki kimechukua maumbo yafuatayo; sikiya, wakamsikiya, unamsikiya na musikiye. Umbo hili la kitenzi linaleta himizo kwa mafunzo ya dini ya Kiislamu. 


\section{Urudiaji wa Neno Moja}

Ni urudiaji uliotumiwa na mtunzi ili kusisitiza na kuonyesha umuhimu wa ujumbe wake. Neno Mola limerudiwa kwa wingi katika Utenzi wa Seyidina Ali na Mudhari bin Darimi ili kuonyesha umuhimu wa Allah katika dini ya Kiislamu. Vilevile neno hili limerudiwa kusisitiza kuwa Mungu ndiye mwanzo tena mwisho na mambo yote katika dini ya Kiislamu huanza naye na pia huisha naye. Yaani yeye ndiye alfa tena omega. Isitoshe, Mungu ndiye hutoa hukumu na msamaha kwa waja wote.

\section{Antistrofia}

Urudiaji wa kiantistrofia umetokea katika Utenzi wa Seyidina Ali na Mudhari bin Darimi mtunzi anapowasilisha ujumbe wake kwa mpangilio wa maneno uliovurugwa. Kwa mfano, katika ubeti wa 175, mtunzi ametumia mpangilio wa maneno uliovurugwa kama vile; dibaji nimefupiza, kwenda mbee sikuweza. Mpangilio huu wa maneno uliovurugwa unamsaidia mtunzi katika kuleta mapigo na mahadhi kwenye utungo huu. Vilevile ni mbinu ambayo mtunzi ametumia ili kupata idadi sawa ya vina na mizani. Ni mtindo ambao umetumiwa kuteka hisia za msomaji na tena kuvuta umakini wake kwa yale yanayosemwa na mtunzi.

\section{Epimoni}

Huu ni urudiaji wa swali lenye maana sawa. Swali niambie asiyo baba wala mama limerudiwa katika ubeti wa 223, 225 na 226 katika Utenzi wa Seyidina Ali na Mudhari bin Darimi. Swali hili linaulizwa na Mudhari bin Darimi kwa Sheikh Ali. Ni swali linalotumiwa na mtunzi ili kusisitiza kuwa kuna wanadamu ambao waliumbwa na Mungu bila kuzaliwa. Swali hili pamoja na majibu yake yanasisitiza kuwa Mungu ana uwezo wa kuumba wanadamu. Hivyo basi, yeye ndiye mwenye uhai. Masimulizi ya majibu ya swali hili yanamwelekeza Muislamu kufahamu kuwa maisha yake yanategemea maamuzi ya Allah. Mbinu hii inasaidia katika kuleta maudhui ya uumbaji na hata wahusika wa kwanza kuumbwa (Adamu na Hawa) pia wanatambulishwa.

\section{Visawe}

Ni urudiaji unaotokea mtunzi anapokuza wazo kwa kutumia maneno mawili tofauti yaliyo na maana sawa. Kwa mfano, katika Utenzi wa Seyidina Ali na Mudhari bin Darimi, mtunzi ametumia maneno latifu na wadudi katika ubeti wa 64 na 65 anaporejelea sifa za Mola. Maneno haya yana maana sawa ya kwamba Mungu ana sifa nyingi zisizo hesabika. Sifa hizi zinasisitizwa ili kumkumbusha Muislamu kuwa Mungu pekee ndiye wa kuabudiwa. maelezo haya yanatumiwa ili kuonyesha ukuu wa Allah pamoja na uwezo wake. Ni mbinu inayoendeleza maudhui ya unyenyekevu na sifo kwa Mwenyezi Mungu.

\section{Haipozesia}

Ni matumizi ya vifungu sambamba vya maneno vyenye nomino na vitenzi kwa mfululizo. Haipozesia imetumiwa na mtunzi wa Utenzi wa Seyidina Ali na Mudhari bin Darimi katika ubeti wa 307. Vifungu Musa hima kainuka na Fimbo yake kaishika vimetumiwa kwa mfululizo ili kuleta mdundo unaovutia katika utenzi huu. Aidha, vifungu hivi vinatuelekeza katika maudhui ya vita takatifu vinavyopiganwa na Musa kwa Kafiri Firauni ili kuwaokoa wana wa Isiraili.

\section{Urudiaji wa Virai vya Kiarabu}

Mtunzi wa Utenzi wa Seyidina Ali na Mudhari bin Darimi ametumia virai vya Kiarabu katika kazi yake. Virai hivi vinamsaidia mtunzi kufikisha ujumbe wake kwa Waislamu. Virai hivi vimetumiwa na mtunzi katika mshororo na wakati mwingine katika ubeti mzima ili kuonyesha kuwa utenzi huu ni wa Kiislamu. Kwa mfano: Rahamani Li Swamadu na Rahimu Rabbu Shahidu (ubeti wa 12). Aidha, virai vya Kiarabu vimetumiwa ili kuonyesha kuwa hakuna lugha nyingine kamilifu inayoweza kutumiwa kufunza dini ya Kiislamu isipokuwa Kiarabu. Kwa sababu Kiarabu ndiyo lugha takatifu iliyotumiwa na Mtume Muhammadi. Vilevile matumizi ya maneno au vifungu vya Kiarabu inaashiria kuwa lugha hii haiwezi kufasiriwa kwa ukamilifu wake katika lugha zingine. 


\section{Urudiaji wa Sentensi}

Urudiaji huu unatokea katika Utenzi wa Seyidina Ali na Mudhari bin Darimi mtunzi anaporudia sentensi mbalimbali ili kusisitiza ujumbe anaouwasilisha. Kwa mfano, sentensi La ilaha illa-Llahu imerudiwa kama njia ya kuwasisitizia Waislamu kuwa hakuna mwingine anayestahili kuabudiwa isipokuwa Allah. Kwa hivyo siku zote Waislamu wanastahili kukumbuka kuwa Mungu ni mmoja na hakutakwepo mwingine kama yeye. Isitoshe, ukuu na ukubwa wa Mungu hauwezi kulinganishwa wala kupigiwa mfano. Sentensi hii inatambulisha Mungu kama mhusika mkuu katika utungo huu na vilevile katika maisha ya Kiislamu kwa ujumla.

\section{Urudiaji wa Kibwagizo Sahili}

Wamitila (2008:487) anasema kuwa kibwagizo sahili kinaporudiwa kwenye utungo, huwa kinamsaidia msomaji kuelewa maana ya kimsingi kwenye utungo. Mtindo huu umetumiwa na mtunzi katika ubeti wa 2, 40 na 46. Katika beti hizi, vifungu vifuatavyo vimetumiwa mwishoni mwa beti: Nimependa kukwambiya, Nimezie kuwambiya na Mukitaka tawambiya. Mtunzi ametumia kibwagizo sahili kwenye beti hizi kama njia ya kusadidia uelewa wa msomaji kuhusu sifa za Mungu na maonyo dhidi ya maisha. Kibwagizo hiki sahili pia kimetumiwa na mtunzi kuendeleza maudhui ya toba na kumkumbusha Muislamu kuwa anasa hupotosha na hata wakati mwingine kuangamiza.

\section{Urudiaji wa Kibwagizo Kikufu}

Kwa mujibu wa Wamitila (2008:488), kibwagizo kikufu ni kibwagizo kinachojenga aina fulani ya mkufu katika ubeti unaomalizika na ule unaofuata. Kibwagizo hiki kinaleta muundo wa kitu kilichofungwa kwa pamoja. Katika Utenzi wa Seyidina Ali na Mudhari bin Darimi, mtunzi ameunda kibwagizo kikufu katika ubeti wa 33 - 56. Kibwagizo hiki kinaletwa na matumizi ya vifungu hivi; Aliteuwa Jaliya, Wane wakashinda piya, Mwenye panda ya fazaa, Mtu kuwata duniya, Zaidi Mitumi piya, Na Saidi Kurashiya, Umati kushufaiya, Rabi kajiteulia, Na Sayidina Aliya, Nimeziye kuwambiya, Mtakapo kusikiya, Zimekwisha kutimiya, Rabbi aliwateuwa, Mariyamu Kamariya, Nuru ikamzagaa.
Vibwagizo vya beti hizi vinakamilishana kimaudhui. Mtunzi anaelezea suala la uumbaji katika beti hizi. Beti hizi zenye vibwagizo vikufu pia zimechangia katika utambulisho wa wahusika tofauti tofauti katika kazi hii.

\section{Urudiaji wa Kibwagizo Changamano}

Kibwagizo changamano kinatokea katika Utenzi wa Seyidina Ali na Mudhari bin Darimi kutokana na uwezo wa mtunzi wa kutumia kibwagizo sahili na kikufu. Vibwagizo hivi vinavyotokea katika ubeti wa 2, 40 na 46 na pia ubeti wa 33 hadi 56 vinachangia katika kuendeleza maudhui ya uumbaji. Vilevile sifa za Mtume Muhammadi zinakuzwa na kuendelezwa kupitia kwayo. Mtume Muhammadi ni kati ya wahusika waliosawiriwa kama wahusika wakuu. Vilevile mhusika huyu ndiye mhusika muhimu zaidi katika dini ya Kiislamu.

\section{Usambamba}

Kwa mujibu wa Swann na Side Wink (1934:57), usambamba hutokea wakati ubeti ule ule unaporudiwa na mtunzi kwa kutumia maneno tofauti au ishara ili kuwasilisha ujumbe katika utenzi. Usambamba pia hurejelewa kama ulinganishi kwa sababu ni aina ya tamathali ya usemi. Tunahoji kuwa kifungu kinachorudiwa huleta msisitizo kwa wazo ambalo huwasilishwa na mtunzi. Miundo sambamba ni kama kiunganishi kilichoundwa kwa sababu miundo hii inaunganisha sentensi nyingi kamilifu.

Urudiaji wa kifungu cha pili humwezesha msomaji kufasiri sentensi haraka au kwa kasi. Kwa sababu urudiaji huu humsaidia msomaji kupunguza uzito wa kufasiri kifungu. Hali hii humwezesha msomaji kuelewa kifungu. Matumizi ya miundo sambamba inamsaidia mtunzi kuvuta hisia mbalimbali za msomaji. Uwepo wa miundo sawa wa kisintaksia wa vifungu unamsaidia msomaji au wasomaji kuweza kutambua wazo au mawazo sawa ambayo hutokea kwa muundo huo.

Kwa mujibu wa de Beaugrande na Dressler (1968:61), urudiaji sambamba ni urudiaji wa muundo wa kisintaksia wenye maudhui. Wataalamu hawa wanasisitiza kuwa usambamba unatumiwa na mtunzi kueleza tukio ambalo linafanana au tukio 
linalofuatana kwa haraka. Mifano ya aina mbalimbali ya usambamba ni pamoja na;

\section{Usambamba wa Kidhamira}

Wamitila (2008:491) anasema kuwa usambamba huu mara nyingi hutokea wakati wazo ambalo linazungumziwa linarudiwa. Katika Utenzi wa Seyidina Ali na Mudhari bin Darimi, usambamba huu wa kidhamira umetumiwa mtunzi anaporudia masuala mbalimbali kuhusu dini ya Kiislamu (ubeti wa 1- 32, 772 - 775). Katika beti hizi, mtunzi anazungumzia mambo tofauti tofauti kuhusu dini ya Kiislamu. Beti hizi pia zimetumiwa kama kitambulisho na tamati ya masuala yanayouhusiana na dini.

Aidha, mtunzi amezungumzia usambamba wa kidhamira kuhusu Siku ya Kiyama (ubeti 21, 640653). Hapa mtunzi anaelezea namna ambavyo watu watakavyohukumiwa na Allah siku za mwisho. Waislamu wametahadharishwa kujiepusha na maovu kwa sababu wataadhibiwa kutokana na maovu hayo. Isitoshe, mtunzi ameshughulikia usambamba wa dhamira kuhusu masuala ya uumbaji. Masimulizi haya yanatokea katika ubeti wa 33 hadi 63. Katika beti hizi, mtunzi anaelezea namna ambavyo Mungu aliumba vitu vingi ila vinne pekee ndivyo vitakatifu. $\mathrm{Ni}$ masimulizi yanayoelezea chanzo cha Waislamu na kuwepo kwa vitu mbalimbali duniani.

\section{Usambamba wa Kifahiwa}

Ni usambamba unaotokea wakati fahiwa ile moja inaporudiwa huku mtunzi akitumia muundo unaofanana wa sentensi. Katika utungo wetu mfano wa usambamba wa kifaiwa unatokea katika ubeti wa 775. Kwa mfano; Ya Allahu ya Kariimu, Ya Allahu tukirimu na Ya Allahu turahamu. Mtunzi ametumia urudiaji huu ili kuendeleza dhamira ya msamaha. Anamsihi Mungu aweze kuwasamehe Waislamu kwa dhambi waliotenda. Aidha, urudiaji huu wa kifahiwa unaleta msisitizo kwa suala hili la msamaha linalozungumziwa. Hii ni kwa sababu mtunzi anaogopa adhabu itakayowapata watu ambao hawajasamehewa dhambi zao siku ya hukumu.

\section{Usambamba wa Kimuundo}

Huu ni urudiaji ambao mtunzi wa Utenzi wa Seyidina Ali na Mudhari bin Darimi ametumia ili kuwekea mkazo ruwaza yenye muundo sawa. Kwa mfano, katika ubeti wa 26-30, mtunzi ametumia ruwaza yenye muundo sawa kuendeleza maudhui ya toba na msamaha. Katika beti hizi, mtunzi anamuomba Mungu kuwa awape hekima ili wafe wakiwa wamejua dini ya Kiislamu. Vilevile anamsihi Mungu awasaidie Waislamu kutubu ili wajue njia zake, sheria zake na pia wamtambue kama mwokozi. Zaidi ya hayo, mtunzi anawaombea wale ambao hawajapata ufunuo wa kujua dini ya Kiislamu ili waweze kutambua dini hiyo kama dini takatifu ambayo itawasaidia katika kufahamu sheria za Allah na hata kuwaelekeza katika matendo mema.

\section{Usambamba wa Kimaana}

Katika urudiaji huu, muundo unaorudiwa unakuza maana ile ile. Aidha, maana hii sawa inaweza ikakuzwa kwa kubadilisha maneno yanayotumiwa kwenye muundo ambao umerudiwa. Kwa mfano, mtunzi wa Utenzi wa Seyidina Ali na Mudhari bin Darimi amerudia muundo wa beti mbalimbali kwa kutumia maneno tofauti tofauti ili kukuza wazo au ujumbe kuhusu Siku ya Kiyama. Masimulizi haya yanatokea katika ubeti wa 21, 640 hadi 653 na ubeti wa 690-691. Urudiaji huu umetumiwa na mtunzi ili kusisitiza ujumbe kuhusu hukumu na vilevile ili kukuza wahusika pamoja na mandhari ya matukio (Peponi na Jehanamu).

\section{Usambamba wa Kitanakuzi}

Mbinu hii hutokea wakati wazo linalokuzwa katika ubeti wa kwanza linapolinganuliwa au kupingwa katika ubeti unaofuata. Katika Utenzi wa Seyidina Ali na Mudhari bin Darimi, mtunzi ametumia mbinu hii ili kulinganisha na kulinganua namna ambavyo Ibilisi anavyopenda maovu huku akichukia haki na mema. Ni masimulizi yanayotolewa katika ubeti wa 557 hadi 590. Ibilisi anakubali kuwa anawapenda sultani mwenye hana maadili mema, Mkafiri, tajiri ambaye hajasadiki, asiye mcha Mungu, mwenye hana moyo mwema, muongo, mnyakuzi wa mali ya mayatima, muovu, mchoyo na mpenda anasa. 
Kwa upande mwingine anayapinga mambo yote mazuri yanayomfurahisha Mungu. Mambo haya yasiyompendeza Ibilisi ni pamoja na; kumcha Mungu, Sultani mwenye maadili, muumini wa dini ya Kiislamu, mtubu, tajiri msadiki, asiyependa vya haramu, mkataa maovu, mkarimu, mwenye amali njema na mfuata dini.

Matumizi ya usambamba huu yanachangia katika kusisitiza ujumbe kuhusu wema na maovu. Vilevile ni mbinu ambayo imetumiwa na mtunzi ili kuwakuza wahusika nguli (Mungu, Mtume Muhammadi, Malaika na Isa) na mkinzani (Ibilisi). Pamoja na hayo, maudhui tofauti tofauti pia yanajengwa na msimulizi. Maudhui haya ni pamoja na tamaa na ubinafsi, maovu na ukarimu.

\section{Usambamba wa Kisanisi}

Usambamba huu unaendelezwa katika Utenzi wa Seyidina Ali na Mudhari bin Darimi kupitia kwa ukuzaji wa wazo fulani kwa kutumia wazo lingine linalofanana nalo. Mtunzi wa utungo huu anaendeleza masuala ya Siku ya Kiyama kwa kutumia suala la toba. Wazo hili linaendelezwa mtunzi anapowashauri Waislamu waweze kutubu kama njia ya kujiepusha na mabaya. Basi wanapofanya hivyo watapewa kibali cha kuenda Peponi Siku ya Kiyama. Aidha, wazo la Siku ya Kiyama pia linaendelezwa kupitia kwa uelewa wa wema na maovu kwa Muislamu. Uelewa wa wema ni msingi wa maisha mazuri ya Peponi baada ya hukumu huku maovu yakichangia maangamio ya Jehanamu.

\section{Usambamba wa Kitarakimu}

Wamitila (2008:493) anasema kuwa ni usambamba unaotokea wakati tarakimu fulani kama vile moja, mbili, tatu na kadhalika inaporudiwa. Vilevile urudiaji huu unatokea wakati neno moja linaloingizwa fahiwa ya kuhesabu na mtunzi linapotumiwa. Usambamba wa kitarakimu umetokea katika Utenzi wa Seyidina Ali na Mudhari bin Darimi mtunzi anapoorodhesha vitu mbalimbali muhimu vilivyoumbwa na Mungu. Masimulizi haya yanatolewa katika ubeti wa 33 hadi 56 mtunzi anaposema kuwa Mungu aliumba vitu vingi ila vinne pekee ndivyo vitakatifu. Vitu hivi ni pamoja na:
Kwanza, Mungu aliwaumba Malaika wanne wenye kheri. Malaika hawa ni pamoja na Jiburili, Mikaili, Isirafili na Aziraili. Mungu aliwaumba mitume wanne. Mitume hawa ni Iburahimu, Musa, Isa na Muhammadi. Isitoshe, Mitume Abubakari, Umari, Athumani na Sayidina Ali ni mitume mashuhuri. Hii ni kwa sababu waliumbwa kama Mitume wa Waislamu. Vilevile majabali manne yaliumbwa na Mungu. Majabali haya ni pamoja na; Jabali la kheri Kafu, Turu, Huda na Arafiya. Miezi minne mitakatifu pia iliumbwa na Mungu. Mwezi wa Muharamu, Rajabu Muhhashamu, Shaabani na Ramadhani.

Zaidi ya hayo Mungu aliumba siku nne takatifu. Siku ya Juma, Idi mbili na Arafa. Mungu aliwatengea Waislamu masiku bora. Masiku haya ni Lailatu I'Haji, idi na Laila Miiraji. Baada ya Mungu kuwaumba wanawake wengi, aliweza kuwateua wanne pekee. Wanawake hawa ni Amina mwana wa Bashira, Mwana Sara mkewe Mtume Ibrahimu, Mariamu bintiye I'Umari na Saidatina ambaye ni bintiye Shafiina.

Matumizi ya usambamba huu inatuelekeza katika chanzo cha viumbe mbalimbali duniani. Aidha, ni masimulizi yanayotuelekeza katika ukuaji wa maudhui ya uumbaji na wahusika wa utungo huu kama vile; Adamu, Hawa, Malaika Jiburili, milima na hata ndege. Vilevile mazingira ya utenzi huu pia tunachorewa na mtunzi kupitia kwa usambamba huu wa kitarakimu. Kwa mfano, mtunzi anatuchorea ulimwengu mtupu unaojazwa na Mungu kwa hatua.

\section{Usambamba wa Kiwizani}

Msokile (1992:120) anafafanua kuwa wizani hurejelea mapigo maalum ambayo yanamsaidia mtunzi katika kujenga uzuri wa utungo wake. Utenzi wa Seyidina Ali na Mudhari bin Darimi una idadi maalum ya mizani iliyopangwa kwa njia maalum. Aidha, mpangilio huu vilevile huambatana na urefu maalum.

Katika utenzi huu, usambamba wa kiwizani, umemsaidia mtunzi kujenga wizani kwa vile ametumia ruwaza maalum inayokaririwa huku sentensi fupi na ndefu zikibadilishwa katika ruwaza za sauti. Mfano ni katika ubeti wa 135B ambao vifungu vifuatavyo vimetumiwa; Basi ndiye tuombao, Moto twepukane nao na Shetani na

106 | This work is licensed under a Creative Commons Attribution 4.0 International License. 
wandamao. Vifungu hivi vina mizani minane katika kila kifungu na sauti ya mwisho inaishia kwa silabi o. Mtindo huu umemsaidia mtunzi katika kueleza maudhui ya toba kwa mdundo maalum. Aidha, ni mtindo unaoleta mvuto kwa yale yanayosemwa na mtunzi. Hii ni kwa sababu yanayosemwa yamewasilishwa kwa mdundo maalum.

\section{Usambamba wa Kimandhari}

Usambamba wa kimandhari hutokea mtunzi anaporudia mazingira maalum ama mazingira yanayokaribia kufanana. Katika Utenzi wa Seyidina Ali na Mudhari bin Darimi, mtunzi amerudia mazingira ya Peponi na Jehanamu kwa wingi. Mazingira haya yamerejelewa mtunzi anapowaelezea Waislamu maisha yatakayowapata baada ya hukumu siku ya mwisho. Mandhari ya Peponi yanarejelewa ili kuonyesha pahali patulivu penye maisha ya kuvutia na watu walionawiri kiafya (ubeti wa 135B, C, D, 141-144 na ubeti wa 640-653, 690-691).

Aidha, yale ya Jehanamu yamerudiwa ili kuashiria pahali penye maangamio huku watu wakichomeka kwenye moto mkali wakiliwa na joka. Isitoshe, miili ya waja Jehanamu imedhoofika kutokana na mateso yasiyoisha. Mbinu hii imetumiwa na mtunzi kukuza mandhari mbalimbali yanayochangia katika kuendeleza maudhui ya utenzi huu. Vilevile tabia za Waislamu pia yanakuzwa kwa kuzingatia maamuzi yatakayotolewa na Mungu baada ya siku ya mwisho.

\section{Hitimisho}

Katika makala haya tumeangazia namna ambavyo urudiaji wa kisarufi unavyochangia ukuaji wa fani na maudhui katika Utenzi wa Seyidina Ali na Mudhari bin Darimi. Katika uhakiki huu, tumedhihirisha jinsi mtunzi ametumia aina mbalimbali za urudiaji wa kisarufi. Aidha, tumebainisha jinsi urudiaji wa kisarufi unavyochangia fani na maudhui wa utungo huu. Kwa mfano, mtunzi anapotumia usambamba wa kitarakimu anarudia nambari nne inayoonyesha ukamilifu katika uumbaji katika dini ya Kiislamu. Nambari hii inapotumiwa maudhui ya uumbaji inatokea kwa sababu mtunzi anatuelezea kuwa Mungu aliumba vitu vingi ila vinne pekee ndivyo vitakatifu katika dini ya Kiislamu. Isitoshe, kupitia kwa uumbaji wa viumbe, tabia za wahusika tofauti tofauti zinakuzwa kutokana na matendo na shughuli zao za kila siku baada ya kuumbwa. Mwisho tumeonyesha athari ya mbinu hii ya urudiaji wa kisarufi katika uwasilishaji wa maana.

\section{MAREJELEO}

Abrams. M. H. (2015). A Glossary of Literary Terms. New York: Cengage Learning, Inc.

de Beaugrande, R. A. \& Dressler, W. U. (1981). Einfuhrung in die TexHinguistick (Konzepte Sprach-Und Literaturwissenschaft28). Tubingen: Niemeyer.

Croft, S. \& Robert, M. (2000). Exploring Language of Literature. London: Oxford University Press.

Leech, G. N. (1969). A Linguistic Guide to English Poetry. London: Longman Group.

Msokile, M. (1992). Kunga za Fasihi na Lugha. Dar es Salaam: Educational Publishers and Distributors Limited.

Swann, R. \& Side Wink, J. (1934). The Making of English Verses. London: Oxford University Press.

Wamitila, K. W. (2003). Kamusi ya Fasihi: Istilahi na Nadharia. Nairobi: Focus publishers. (2008). Kanzi ya Fasihi 1: Msingi wa Uchanganuzi wa Kiswahili wa Fasihi. Nairobi: Vide- Muwa Publishers Ltd.

Wamutiso, K. (2014). "Number Symbolism (nos. 1, 4, 7 and 1,000) in Swahili Poetry: The case of Utenzi wa Fatumah", Kiswahili Vol. 77, pp. 132148. 